\title{
Estilos de Aprendizaje y Rendimiento Académico en Estudiantes de la Universidad María Auxiliadora.
}

\author{
Learning Styles and Academic Performance in college students of University Maria Auxiliadora
}

Jhonatan Ponce Cumbreras ${ }^{1}$ y Carlos Gamarra Bustillos ${ }^{2}$

\section{RESUMEN}

Objetivo: Comprobar si existe relación entre los estilos de aprendizaje y el rendimiento académico en los estudiantes de la Universidad María Auxiliadora. Materiales y Métodos: Estudio descriptivo correlacional, la población estuvo conformada por 650 alumnos que culminaron el semestre 2014-I, de las carreras profesionales de Administración de Negocios Internacionales, Contabilidad y Finanzas, Marketing Empresarial, Farmacia y Bioquímica y Enfermería de la Universidad María Auxiliadora, el tamaño de la muestra estuvo conformada por 142 alumnos que culminaron el semestre 2014-I. Se utilizó el Cuestionario de Honey y Alonso de Estilos de Aprendizaje (CHAEA) y el promedio ponderado para medir el rendimiento académico de los alumnos. Resultados: El rendimiento académico de las mujeres tiene correlación con el estilo pragmático en estudiantes de la UMA, con un coeficiente de correlación significativa a 0,027. El rendimiento académico de los estudiantes de la carrera profesional de Administración de Negocios Internacionales tiene correlación con el estilo activo; el rendimiento académico de los estudiantes de la carrera profesional de Contabilidad y Finanzas tiene correlación con el estilo teórico; el rendimiento académico de los estudiantes de la carrera profesional de Marketing Empresarial tiene correlación con el estilo reflexivo y el rendimiento académico de los estudiantes de la carrera profesional de Enfermería tiene correlación con el estilo activo, siendo los coeficientes de correlación significativa de 0,025 - 0,038 - 0,027 -0,016 respectivamente. Conclusión: En términos generales el rendimiento académico de los estudiantes de la Universidad María Auxiliadora, Semestre 2014-I, expresado en el promedio ponderado de las notas de los estudiantes, presenta una correlación significativa con el estilo de aprendizaje pragmático.

PALABRA CLAVE: Estilo de aprendizaje - Rendimiento académico

\section{ABSTRACT}

Objective: To determine whether there is a relationship between learning styles and academic achievement in students of the University of Mary Help. Materiales and Methods: correlational descriptive study population consisted of 650 students who completed the semester 2014-I, of the careers of International Business Administration, Accounting and Finance, Business Marketing, Pharmacy and Biochemistry and Nursing at the University of Mary Help The sample size consisted of 142 students who completed the semester 2014-I. Questionnaire Honey and Alonso Learning Styles (CHAEA) and the weighted average was used to measure the academic performance of students. Results: The academic performance of women correlates with the pragmatic style UMA students, with a coefficient of correlation significant at 0.027 . The academic performance of students in the career of International Business Administration correlates with the active style; the academic performance of students of the career of Accounting and Finance is correlated with the theoretical style; the academic performance of students of the career of Business Marketing correlates with reflective style and academic performance of students in the career of Nursing correlates with the active style, with significant correlation coefficients of 0.025 to $0.038-0.027$ to 0.016 respectively. Conclusion: Overall, the academic performance of students of the University of Mary Help Semester 2014-I, expressed as the weighted average of student grades, presents a significant correlation with the style of pragmatic learning.

KEYWORDS: Learning style - Academic Performance

\footnotetext{
$\mathbf{1}$ Psicólogo egresado de la Facultad de Psicología, Universidad Nacional Mayor de San Marcos

${ }^{2}$ Licenciado en Enfermería, Magister en Gestión y Planeamiento Educativo, Doctorado en Salud Publica.

Universidad Nacional Mayor de San Marcos. Lima 01. Perú.
} 


\section{INTRODUCCIÓN}

Los estudios realizados en relación a los estilos de aprendizaje en los estudiantes se originan en los años cuarenta y cincuenta del siglo pasado, los cuales se enfocaron en los estilos cognitivos, posteriormente en los años setenta, además de los estilos cognitivos surge una nueva inquietud en los educadores y pedagogos, que es el cómo aprenden los estudiantes (Ortiz y Canto, 2013).

El concepto estilo se emplea en psicología como la forma particular en la que cada individuo realiza una actividad, lo cual se refleja, por ejemplo, a un estilo de comunicación, estilo de vida o estilo de hablar (Aguilera y Ortiz, 2009, en Ortiz y Canto, 2013). Además, se puede concluir que cada persona tiene una manera particular de hacer determinadas funciones o actividades, es decir puede tener un estilo de hablar, pensar o reír. De manera similar cuando se aprende algo el sujeto privilegia el estilo que se le acomode sobre otros $\mathrm{y}$ en un determinado momento puede combinarlos dependiendo de la tarea (Ortiz y Canto, 2013).

Los estilos de aprendizaje vienen a ser aquellas variables personales que se encuentran entre la inteligencia y la personalidad, dichos estilos van a determinar las diversas formas en que se aborda, planifica y responde a las diferentes demandas del aprender, es así que desde el punto de vista de la teoría del aprendizaje experiencial y de su desarrollo, se puede clasificar en cuatro diferentes a los estilos de aprendizaje, esto partiendo de las diferencias individuales de acceso al conocimiento (Alonso, Gallego y Honey, 1995, en Camarero, Martín y Herrero, 2000):

Estilo de Aprendizaje Activo, es animador, improvisador, descubridor, arriesgado, espontáneo; aprende mejor cuando se lanza a una actividad que le presente un desafío, realiza actividades cortas y de resultado inmediato $\mathrm{y}$ cuando hay emoción, drama y crisis. Le cuesta mucho aprender cuando tienen que adoptar un papel pasivo, cuando tiene que asimilar, analizar e interpretar datos y cuando tiene que trabajar solo.

Estilo de Aprendizaje Reflexivo, es ponderado, concienzudo, receptivo, analítico y exhaustivo. Aprende mejor cuando adopta la postura de observador, ofrece observaciones y analiza la situación, y cuando piensa antes de actuar. Además presenta dificultades para aprender al obligársele a convertirse en el centro de la atención y si tiene que actuar sin poder planificar previamente.

Estilo de Aprendizaje Teórico, es metódico, lógico, objetivo, crítico y estructurado. Aprende mejor a partir de modelos, teorías, sistemas con ideas y conceptos que presenten un desafío y cuando tiene oportunidad de preguntar e indagar. Y posee dificultades para aprender con actividades que impliquen ambigüedad e incertidumbre, cuando tiene que actuar y $\sin$ fundamento teórico.

Estilo de Aprendizaje Pragmático, es experimentador, práctico, directo, eficaz y realista. Aprende mejor cuando relaciona actividades teóricas con prácticas, si ve a los demás hacer algo, y si tiene la posibilidad de poner en práctica inmediatamente lo que ha aprendido. Además le cuesta trabajo aprender cuando no se relaciona con sus necesidades inmediatas $y$ cuando lo que hace no está relacionado con la realidad.

\section{MATERIAL Y MÉTODOS}

\section{Nivel y Tipo de Investigación}

El tipo de investigación fue el tipo Correlacional, ya que se tuvo como propósito describir, analizar e interpretar la relación entre las variables estilos de aprendizaje y rendimiento académico en una situación determinada (Sánchez y Reyes, 2006). El nivel de Investigación realizado fue el Descriptivo, ya que el estudio describe constructos existentes, cuales requieren ser estudiados una circunstancia temporo-espacial determinada (Sánchez y Reyes, 2006).

\section{Diseño de Investigación}

La investigación se realizó en dos etapas. En primer lugar se utilizó el diseño correlacional simple (Sánchez y Reyes, 2006), en el cual se estudiaron las relaciones entre los Estilos de Aprendizaje, del Cuestionario de Honey y Alonso de Estilos de Aprendizaje (CHAEA), y el rendimiento académico, por medio del promedio ponderado de los alumnos. En segundo lugar se empleó el diseño descriptivo simple (Sánchez y Reyes, 2006), para identificar los niveles de los estilos de aprendizaje y sus dimensiones que predominan en los estudiantes de la Universidad María Auxiliadora. 


\section{Participantes}

La población estuvo conformada por 650 alumnos de las diferentes carreras profesionales de la Universidad María Auxiliadora, varones y mujeres, cuyas edades oscilan entre los 15 y 61 años de edad. El tamaño de la muestra estuvo conformada por 142 estudiantes de las carreras profesionales de Administración de Negocios Internacionales, Contabilidad y Finanzas, Marketing Empresarial, Farmacia y Bioquímica, y Enfermería, varones y mujeres, cuyas edades oscilan entre los 17 y 45 años de edad, de la Universidad María Auxiliadora.

\section{Instrumento}

El instrumento empleado fue el Cuestionario de Honey y Alonso de Estilos de Aprendizaje (CHAEA) que mide el estilo de aprendizaje en relación a los aportes teóricos de Kolb (1984) y Heny y Mumford (1986), los cuales coinciden en que el desarrollo del proceso de aprendizaje es cíclico y se da en cuatro etapas, en las que influyen las experiencias vividas, el medio ambiente y lo heredado. (Escurra, 2011). Este instrumento está compuesto por 80 ítems presentados en forma de preguntas, de las cuales 20 corresponden a cada uno de los estilos de aprendizaje.

\section{Procedimiento de recolección de datos}

El procedimiento de recolección de datos, se llevó a cabo en la Universidad María Auxiliadora, en una primera etapa se aplicó el cuestionario CHAEA; y en una segunda etapa se recolecto la información sobre el rendimiento académico de los estudiantes, por medio de sus promedios ponderados obtenidos.

\section{RESULTADOS}

La tabla 1, muestra la distribución de estudiantes de la Universidad María Auxiliadora pertenecientes al semestre 2014-I, según los estilos de aprendizaje; evidenciando que la mayor proporción de los estudiantes presenta un nivel

\section{Análisis Estadístico}

En relación a los estadísticos descriptivos, se usó la media aritmética, que es una medida de tendencia central y descriptiva, (Hernández, Fernández y Baptista, 2010), y con ella se conoció los puntajes medios de la muestra en relación a los niveles de los estilos de aprendizaje. Por otra parte, para la comprobación de las hipótesis, se realizó el análisis estadístico inferencial, que en un primer momento se efectuó a través de un análisis o prueba de normalidad a través de la Prueba de Bondad de ajuste a la curva normal de Kolmogorv-Smirnov, que tiene por finalidad establecer si los datos de una muestra proceden de una población con distribución normal (Hernández, Fernández y Baptista, 2010), con la cual se conoció que la muestra no se aproxima a una distribución normal. Es por ello que en la comprobación de las hipótesis se empleó las pruebas no paramétricas, y debido a que se trata de correlacionar una muestra en función a dos constructos, es que se empleó la rho de Sperman que se emplean en variables en un nivel de medición ordinal, en los cuales los casos se ordenan según rangos (Hernández, Fernández, y Baptista, 2010).

Para los cálculos estadísticos de las muestras, se utilizó un nivel de significación de 0.05. Además se empleó el programa estadístico SPSS 21.0 IBM para Windows, con el que se realizó todo el proceso de análisis estadístico de los datos.

promedio de estilo de aprendizaje activo (63.4\%), seguido por el estilo de aprendizaje teórico (54.2\%), estilo de aprendizaje pragmático (50.0\%) y un nivel alto de estilo de aprendizaje reflexivo $(45.1 \%)$. 
Tabla 1. Estilos de aprendizaje de los estudiantes de la Universidad María Auxiliadora, Semestre 2014-I.

\begin{tabular}{|c|c|c|c|c|c|c|c|c|}
\hline \multirow{2}{*}{ Niveles } & \multicolumn{2}{|c|}{ Estilo Activo } & \multicolumn{2}{|c|}{ Estilo Reflexivo } & \multicolumn{2}{|c|}{ Estilo Teórico } & \multicolumn{2}{|c|}{$\begin{array}{c}\text { Estilo } \\
\text { Pragmático }\end{array}$} \\
\cline { 2 - 9 } & $\mathbf{N}$ & $\%$ & $\mathbf{N}$ & $\%$ & $\mathbf{N}$ & $\%$ & $\mathbf{N}$ & $\%$ \\
\hline Bajo & 23 & $16.2 \%$ & 52 & $36.6 \%$ & 26 & $18.3 \%$ & 9 & $6.3 \%$ \\
\hline Promedio & 90 & $63.4 \%$ & 26 & $18.3 \%$ & 77 & $54.2 \%$ & 62 & $43.7 \%$ \\
\hline Alto & 29 & $20.4 \%$ & 64 & $45.1 \%$ & 39 & $27.5 \%$ & 71 & $50.0 \%$ \\
\hline Total & 142 & $100.0 \%$ & 142 & $100.0 \%$ & 142 & $100.0 \%$ & 142 & $100.0 \%$ \\
\hline
\end{tabular}

Tabla 2. Análisis de normalidad de los estilos de aprendizaje de los estudiantes de la Universidad María Auxiliadora, Semestre 2014-I.

\begin{tabular}{|l|c|c|c|}
\cline { 2 - 4 } \multicolumn{1}{c|}{} & Estadístico & $\mathbf{N}$ & $\mathbf{p}$ \\
\hline Estilo de Aprendizaje Activo & .226 & 142 & .000 \\
\hline Estilo de Aprendizaje Reflexivo & .174 & 142 & .000 \\
\hline Estilo de Aprendizaje Teórico & .193 & 142 & .000 \\
\hline Estilo de Aprendizaje Pragmático & .208 & 142 & .000 \\
\hline
\end{tabular}

$\mathrm{p}<.05$

La tabla 3, establece el nivel de correlación entre el rendimiento académico y los estilos de aprendizaje de estudiantes de la Universidad María Auxiliadora, pertenecientes al semestre 2014-I; evidenciando que el rendimiento académico tiene correlación con el estilo pragmático a nivel general en estudiantes de la UMA, con un coeficiente de correlación significativa a 0,05 .

Tabla 3. Correlación entre el rendimiento académico y los estilos de aprendizaje de los estudiantes de la Universidad María Auxiliadora, Semestre 2014-I.

\begin{tabular}{|c|c|c|c|c|c|}
\hline & & $\begin{array}{l}\text { Estilo } \\
\text { Activo }\end{array}$ & $\begin{array}{c}\text { Estilo } \\
\text { Reflexivo }\end{array}$ & $\begin{array}{c}\text { Estilo } \\
\text { Teórico }\end{array}$ & $\begin{array}{c}\text { Estilo } \\
\text { Pragmático }\end{array}$ \\
\hline \multirow{2}{*}{$\begin{array}{l}\text { Rendimiento } \\
\text { académico }\end{array}$} & $\begin{array}{l}\text { Coeficiente de } \\
\text { correlación }\end{array}$ & $\begin{array}{c}- \\
.075\end{array}$ & .063 & .273 & $.050 *$ \\
\hline & Sig. (bilateral) & .374 & .459 & .001 & .543 \\
\hline
\end{tabular}

*Correlación significativa a .05 
La tabla 4, establece el nivel de correlación entre el rendimiento académico según sexo y los estilos de aprendizaje de estudiantes de la Universidad María Auxiliadora, pertenecientes al semestre 2014-I; evidenciando que el rendimiento académico de las mujeres tiene correlación con el estilo pragmático en estudiantes de la UMA, con un coeficiente de correlación significativa a 0,027 ; mientras tanto en varones no se muestra ninguna correlación significativa con alguno de los estilos de aprendizaje.

Tabla 4. Correlación entre rendimiento académico según sexo y los estilos de aprendizaje de los estudiantes de la Universidad María Auxiliadora, Semestre 2014-I.

\begin{tabular}{|c|c|c|c|c|c|}
\hline \multicolumn{2}{|l|}{$\begin{array}{l}\text { Rendimiento } \\
\text { Académico según sexo }\end{array}$} & $\begin{array}{c}\text { Estilo } \\
\text { Activo }\end{array}$ & $\begin{array}{c}\text { Estilo } \\
\text { Reflexivo }\end{array}$ & $\begin{array}{c}\text { Estilo } \\
\text { Teórico }\end{array}$ & $\begin{array}{c}\text { Estilo } \\
\text { Pragmático }\end{array}$ \\
\hline \multirow{2}{*}{ Varones } & $\begin{array}{c}\text { Coeficiente de } \\
\text { correlación }\end{array}$ & .138 & .181 & .256 & .118 \\
\cline { 2 - 6 } & Sig. (bilateral) & .424 & .291 & .131 & .492 \\
\hline \multirow{2}{*}{ Mujeres } & $\begin{array}{c}\text { Coeficiente de } \\
\text { correlación }\end{array}$ & -.139 & $.034^{*}$ & .285 & $.027^{*}$ \\
\cline { 2 - 6 } & Sig. (bilateral) & .155 & .733 & .003 & .783 \\
\hline
\end{tabular}

*Correlación significativa a .05

La tabla 5, muestra el nivel de correlación entre el rendimiento académico según carrera profesional y los estilos de aprendizaje de estudiantes de la Universidad María Auxiliadora, pertenecientes al semestre 2014-I; evidenciando que el rendimiento académico de los estudiantes de la carrera profesional de Administración de Negocios Internacionales tiene correlación con el estilo activo; el rendimiento académico de los estudiantes de la carrera profesional de
Contabilidad y Finanzas tiene correlación con el estilo teórico; el rendimiento académico de los estudiantes de la carrera profesional de Marketing Empresarial tiene correlación con el estilo reflexivo y el rendimiento académico de los estudiantes de la carrera profesional de Enfermería tiene correlación con el estilo activo, siendo los coeficientes de correlación significativa de $0,025-0,038-0,027-0,016$ respectivamente.

Tabla 5. Correlación entre rendimiento académico según carrera profesional y los estilos de aprendizaje, en los estudiantes de la Universidad María Auxiliadora, Semestre 2014-I.

\begin{tabular}{|c|c|c|c|c|c|}
\hline \multicolumn{2}{|l|}{$\begin{array}{l}\text { Rendimiento } \\
\text { Académico según } \\
\text { carrera profesional }\end{array}$} & $\begin{array}{c}\text { Estilo } \\
\text { Activo }\end{array}$ & $\begin{array}{c}\text { Estilo } \\
\text { Reflexivo }\end{array}$ & $\begin{array}{c}\text { Estilo } \\
\text { Teórico }\end{array}$ & $\begin{array}{c}\text { Estilo } \\
\text { Pragmático }\end{array}$ \\
\hline $\begin{array}{c}\text { Administración de } \\
\text { Negocios } \\
\text { Internacionales }\end{array}$ & $\begin{array}{c}\text { Coeficiente de } \\
\text { correlación }\end{array}$ & $.025^{*}$ & .232 & .308 & .055 \\
\cline { 2 - 6 } & Sig. (bilateral) & .880 & .156 & .057 & .740 \\
\hline $\begin{array}{c}\text { Contabilidad y } \\
\text { Finanzas }\end{array}$ & $\begin{array}{c}\text { Coeficiente de } \\
\text { correlación }\end{array}$ & .184 & -.162 & $.038^{*}$ & .125 \\
\cline { 2 - 6 } Marketing & Sig. (bilateral) & .479 & .534 & .884 & .633 \\
\hline \multirow{2}{*}{ Empresarial } & $\begin{array}{c}\text { Coeficiente de } \\
\text { correlación }\end{array}$ & -.727 & $-.027^{*}$ & -.112 & .112 \\
\cline { 2 - 6 } & Sig. (bilateral) & .027 & .945 & .774 & .774 \\
\hline
\end{tabular}




\begin{tabular}{|c|c|c|c|c|c|}
\hline \multirow{2}{*}{$\begin{array}{c}\text { Farmacia y } \\
\text { Bioquímica }\end{array}$} & $\begin{array}{c}\text { Coeficiente de } \\
\text { correlación }\end{array}$ & -.280 & -.199 & .120 & .156 \\
\cline { 2 - 6 } & Sig. (bilateral) & .076 & .213 & .455 & .331 \\
\hline \multirow{2}{*}{ Enfermería } & $\begin{array}{c}\text { Coeficiente de } \\
\text { correlación }\end{array}$ & $-.016^{*}$ & .352 & .384 & .119 \\
\cline { 2 - 6 } & Sig. (bilateral) & .925 & .035 & .021 & .488 \\
\hline
\end{tabular}

*Correlación significativa a .05

\section{DISCUSIÓN}

El análisis de los resultados del presente estudio, indican que el estilo de aprendizaje pragmático tiene una correlación positiva con el rendimiento académico de los estudiantes de la Universidad María Auxiliadora, lo que se contrapone a todas las investigaciones anteriormente realizadas, ya que en algunas se señala que son los estilos teórico y activo los que presentan esta influencia (Blumen, Rivero y Guerrero, 2011), en otro estudio el activo y reflexivo (Esguerra y Guerrero, 2009, Sanabria, 2009), o solo el reflexivo (Ruiz, Trillos y Morales, 2006); pero en ninguno de ellos está la presencia del estilo pragmático.

Los resultados encontrados en el presente estudio, sugieren que el estilo de aprendizaje estaría relacionado a la cultura académica de formación en primaria y secundaria, es decir, que el sistema educativo de cada país o región presenta una determinada metodología de enseñanza que da surgimiento a un estilo de aprendizaje definido; si a nivel de estudios superiores se emplea dicho estilo de aprendizaje formado en los estudiantes, entonces se podrá obtener un mejor rendimiento académico, tal como lo señalan Ortiz y Canto (2013).

Es así que se llega a determinar, que en general los estudiantes de la Universidad María Auxiliadora, son experimentadores, prácticos, directos, eficaces y realistas, los cuales aprenden mejor cuando ponen en práctica inmediatamente lo aprendido en teoría, pero les cuesta mucho trabajo aprender con aquellas actividades que no tienen una finalidad aparente a su realidad (Camarero, Martín, Herrero, 2000).

\section{CONCLUSION}

El rendimiento académico de las mujeres tiene correlación con el estilo pragmático en estudiantes de la UMA, con un coeficiente de correlación significativa a 0,027 ; mientras tanto en varones no hay correlación significativa con alguno de los estilos de aprendizaje.

El rendimiento académico de los estudiantes de la carrera profesional de Administración de Negocios Internacionales tiene correlación con el estilo activo; el rendimiento académico de los estudiantes de la carrera profesional de Contabilidad y Finanzas tiene correlación con el estilo teórico; el rendimiento académico de los estudiantes de la carrera profesional de Marketing Empresarial tiene correlación con el estilo reflexivo y el rendimiento académico de los estudiantes de la carrera profesional de Enfermería tiene correlación con el estilo activo, siendo los coeficientes de correlación significativa de 0,025-0,038-0,027-0,016 respectivamente.

En términos generales el rendimiento académico de los estudiantes de la Universidad María Auxiliadora, Semestre 2014-I, expresado en el promedio ponderado de las notas de los estudiantes, presenta una correlación significativa con el estilo de aprendizaje pragmático.

\section{AUTOR DE CORRESPONDENCIA:}

Carlos Gamarra, Profesor Universidad María Auxiliadora.

Av. Canto Bello 431- Ciudad Universitaria, Lima 36 - Perú.

Teléfono: +51-1-3891212.

E-mail: cgbenfermero@ hotmail.com 


\section{REFERENCIAS BIBLIOGRAFICAS}

1. Blumen, S., Rivero, C., y Guerrero, D. (2011). Universitarios en educación a distancia: estilos de aprendizaje y rendimiento académico. Revista de Psicología, 29(2), pp. 226-243. Pontificia Universidad Católica del Perú.

2. Camarero, F., Martín, F., y Herrero, J. (2000). Estilos y estrategias de aprendizaje en estudiantes universitarios. Psicothema, 12(4), pp. 615-622. Universidad de Oviedo: España.

3. Escurra, M. (2011). Análisis psicométrico del Cuestionario de Honey y Alonso de Estilos de Aprendizaje (CHAEA) con los modelos de la Teoría Clásica de los Tests y de Rasch. Persona, 14, pp. 71-109. Universidad de Lima.

4. Esguerra, G., y Guerrero, P. (2010). Estilos de aprendizaje y rendimiento académico en estudiantes de Psicología. Diversitas: Perspectivas en Psicología, 6(1), pp. 97-109. Universidad Santo Tomás: Colombia.

5. Hernández, R., Fernández, C., y Baptista, P. (2010). Metodología de la Investigación. México: Mc Graw Hill.

6. Ortiz, A., y Canto, P. (2013). Estilos de aprendizaje y rendimiento académico en estudiantes de Ingeniería en México. Revista Estilos de Aprendizaje, 11(11), pp. 160-177.
7. Ruiz, B., Trillos, J., y Morales, J. (2006). Estilos de aprendizaje y rendimiento académico en estudiantes universitarios. Revista Galeco-Portuguesa de Psicoloxía e Educación, 11-12(13), pp. 441-457. Universidad Tecnológica de Bolívar: Colombia.

8. Sanabria, N. (2009). Relación entre los estilos de aprendizaje y el rendimiento académico en estudiantes universitarios. Tesis para optar al Título de Psicólogo. Universidad Pontificia Bolivariana.

9. Sánchez, H., y Reyes, C. (2006). Metodología y diseños en la investigación científica. Lima: Editorial Visión Universitaria.

Recibido: 09/04/2015

Aceptado: 30/04/2015 